\title{
Erratum to: Applied Computer Science for GGOS Observatories
}

\author{
Alexander N.J. Neidhardt
}

\section{Erratum to}

A. Neidhardt, Applied Computer Science for GGOS Observatories, Springer Textbooks in Earth Sciences, Geography and Environment, DOI 10.1007/978-3-319-40139-3

In the original version, the author name was given as Alexander Neidhardt without the middle initials throughout the book.

The correct version of the author name is Alexander N.J. Neidhardt (with middle initials).

The updated original online version of the original book can be found under DOI 10.1007/978-3-319-40139-3 